\title{
Sentidos del cuidado en centros de privación de libertad para adolescentes en Uruguay
}

\author{
Cecilia Montes-Maldonado, Mg. \\ Profesora Universidad de la República, Uruguay \\ y Universidad Autónoma de Barcelona*
}

cmontes@psico.edu.uy

\section{Resumen (analítico)}

El artículo explora sentidos y significados asociados al cuidado por parte de agentes responsables de la custodia de adolescentes en centros de privación de libertad en Uruguay. La investigación cualitativa es un estudio de caso de tipo único, descriptivo y en profundidad que presenta un corpus compuesto por entrevistas, documentos, observaciones y registro en diario de campo. Se describen tres categorías de análisis en las que se reflexiona sobre el cuidado: acerca de la medida socioeducativa, la seguridad como criterio de funcionamiento y el acceso a los derechos a través de la política punitiva. Se concluye que existen visiones plurales asociadas al cuidado y que el tratamiento de los y las adolescentes se basa en el castigo por la vía del encierro disciplinante que legitima el uso de prácticas violentas.

\section{Palabras clave}

Delincuencia juvenil, cuidados, investigación social.

\section{Tesauro}

Tesauro de Ciencias Sociales de la Unesco.
Para citar este artículo

Montes-Maldonado, C. (2019). Sentidos del cuidado en centros de privación de libertad para adolescentes en Uruguay. Revista Latinoamericana de Ciencias Sociales, Niñez y Juventud, 17(2), 1-22. doi: 10.11600/1692715x.17216

Historial

Recibido: 12.02.2019

Aceptado: 20.03.2019

Publicado: 30.06 .2019

Información artículo

El presente artículo corresponde a una investigación realizada para la tesis del Doctorado Interuniversitario en Estudios de Género: Culturas, Sociedades y Políticas de la Universidad Autónoma de Barcelona (3 de setiembre, 2016-3 de octubre, 2019). Financiación: Beca de Posgrado en el Exterior, Agencia Nacional de Investigación e Innovación, Uruguay. Área: Ciencias Sociales. Subárea: Interdisciplinaria. 


\section{Meanings of care in adolescent detention centers in Uruguay}

\section{Abstract (analytical)}

This article analyzes the meanings and ideas associated with care among the adults who operate adolescent detention centers in Uruguay. This qualitative research involves a unique, descriptive and in-depth case study that presents a corpus consisting of interviews, documents, observations and field notes. Three categories of analysis are described in relation to the concept of care: care as a socio-educational measure, safety as an operating standard and access to rights through punitive politics. The article argues that there are pluralist visions associated with care and the treatment of adolescents that are based on punishment through disciplinary confinement, which legitimizes the use of violent practices.

\section{Keywords}

Juvenile delinquency, adolescence, care, institutionalization, social research

\section{Significado dos cuidados em centros de detenção juvenil no Uruguay}

\section{Resumo (analítico)}

O artigo explora significados e ideias associadas ao cuidado dos agentes responsáveis pela custódia dos adolescentes nos centros de privação de liberdade no Uruguai. A pesquisa qualitativa é um estudo de caso único, descritivo e aprofundado, que apresenta um escopo composto por entrevistas, documentos, observações e anotações de diário de campo. Três categorias de análise são descritas nas quais se consideram o cuidado: a medida socioeducativa, a segurança como critério de funcionamento e o acesso aos direitos por meio de políticas punitivas. Conclui-se que existem visões plurais associadas ao cuidado e que o tratamento dos adolescentes é baseado na punição por meio do confinamento disciplinar que legitima o uso de práticas violentas.

\section{Palavras-chave}

Delinquência juvenil; adolescência; cuidado; institucionalização; pesquisa social.

\section{Información autora}

[*] Licenciada en Psicología (2010), Magíster en Psicología Social (2015), Universidad de la República. Profesora e investigadora Facultad de Psicología (UdelaR). Candidata a Doctora en Estudios de Género: Culturas, Sociedades y Políticas por la Universidad Autónoma de Barcelona. Investigadora asociada Sistema Nacional de Investigadores (SNI-ANII). Líneas de investigación: Género, Cuidados, Infancia-Adolescencia. Orcid: 0000-0002-2516-4488. Índice H5: 2. Correo electrónico: cmontes@psico.edu.uy 


\section{Introducción}

Uruguay se alinea con la tendencia regional y mundial del auge punitivo por medio de la ampliación del control y alcance de los sistemas penales sobre la población en general y la población joven en particular. Ejemplos de esto son el endurecimiento de las penas, propuestas de bajar la edad de imputabilidad penal, conformación de nuevos delitos, altas tasas de encarcelamiento, entre otras (Garland, 2018; Fassin, 2018).

En las primeras décadas del siglo XX se institucionaliza en Uruguay la intervención estatal al problema de la infracción de adolescentes y a la situación de desprotección familiar de niños, niñas y adolescentes. Este proceso se materializa con la creación del Código del Niño de 1934 signado por el paradigma de la situación irregular de la infancia. Con el crecimiento de la intervención estatal en este campo y un proceso de mayor normalización de la infancia y adolescencia por medio de la puesta en juego de procedimientos especializados, se continúa hacía el modelo tutelar. Estos modelos contribuyen a la construcción de estereotipos negativos sobre la categoría de adolescente y asocian adolescencia con actividad delictiva. Es con la creación del Código de la Niñez y la Adolescencia (CNA) (Uruguay, 2004), el cual incluye las prerrogativas de la Convención de los Derechos del Niño (Uruguay, 199o), que comienza una lenta expansión de las orientaciones de la doctrina de la protección integral en nuestro país (Leopold, 2014).

Las políticas públicas de infancia y adolescencia desarrolladas brindan protección en las diferentes áreas de la vida. Se han implementado políticas que responden a diseños como modelos de políticas universales del Estado de Bienestar social, políticas sociales tercerizadas o privatizadas, focalizadas y de blindaje social (Laurnaga, 2007). Si bien se ha intentado la universalización de las políticas dirigidas a la infancia y adolescencia, se puede ver como las mismas se conducen a las situaciones definidas como de riesgo, vinculadas al paradigma de la situación irregular de esta población (García, 2010; Medan, 2017) y que responden a políticas de individuación o activación individual definidas por Merklen (2013). 
Entre los términos binarios de protección-control o educación-castigo se erige la ejecución de las medidas privativas de libertad para adolescentes tanto en Uruguay como en la región. A través del disfraz educativo de las medidas judiciales, lo que vemos es la primacía del cariz punitivo y de encierro (Ávila-Navarrete, 2017; Cienfuegos, 2015; Leal, \& Macedo, 2019; Medan, Villalta, \& Llobet, 2019). Y los efectos negativos de la privación de libertad para adolescentes a raíz de las diferentes vulneraciones de derechos que provocan (Redondo \& Martínez, 2013; Redondo, Martínez, \& Pueyo, 2012; Tenenbaum, 2017).

La noción de castigo no es unívoca ni lineal; seguimos los aportes foucaultianos, de la sociología del castigo y la criminología crítica que lo definen como una función social compleja. Así Foucault $(2002,2016)$ habla del castigo como una función del poder, instrumental y estratégica que administra los ilegalismos y crea diferencias. Por su parte, Garland (2006) enfatiza el castigo como institución social, organizada y estructurada para expresar el poder del Estado. Implica una suma de factores y no puede verse desde una sola perspectiva. Tiene potencial simbólico y, además, se encuentra influido por la sensibilidad del contexto, la cultura y condicionantes político-económicos. Por último, Baratta (2004) da cuenta de la distribución desigual de los castigos en función de los posicionamientos sociales, jerarquías e intereses.

\section{Sistema Penal Juvenil Uruguayo (SPJU)}

El SPJU abarca los ámbitos, policial, judicial y de ejecución de las medidas judiciales amparado en el CNA (Uruguay, 2004). Las infracciones penales se dividen en graves y gravísimas y conceden responsabilidad a los/as adolescentes de entre 13-17 años pasibles de procedimiento judicial por la infracción cometida. Las medidas judiciales, llamadas socioeducativas, que se adoptan luego del proceso son privativas o no privativas de libertad. La gestión de las mismas está cargo del Instituto Nacional de Inclusión Social Adolescente (Inisa) desde 2016, momento en que se organiza para cumplir con las medidas judiciales y cautelares dictadas por el poder judicial a través de programas oficiales o en convenio con organizaciones de la sociedad civil.

Los centros de privación de libertad son 13 y se encuentran en Montevideo, Canelones y Lavalleja, donde dividen la población según sexo, edad o situación de progresividad en el sistema. Organizados evidenciando características de instituciones totales (Goffman, 2001), cuentan con centros destinados a: ingreso, estudio y derivación; medidas cautelares; máxima contención; jóvenes mayores de 18 años; adolescentes mujeres 
(un único establecimiento); adolescentes varones (1 centro menores de 14 años y varios centros para mayores de 15-17 años), y un centro de semilibertad.

La puesta en marcha del CNA (Uruguay, 2004) fue un paso fundamental en el reconocimiento de los derechos y garantías para niños, niñas y adolescentes que debiera funcionar de impulso para la mejora de sus condiciones de vida. Sin embargo, referentes en la temática (González, 2015, 2016) muestran la comprometida situación de la justicia penal juvenil, especialmente, a partir de la aprobación de diversas modificaciones al CNA (Uruguay, 2004) que contravienen la normativa internacional y que han situado a los/as adolescentes que transitan por alguna esfera del sistema en condiciones inadmisibles.

En esta línea se ubican las principales áreas de conflicto que enfrenta el SPJU, identificadas por investigaciones que muestran las condiciones de privación de libertad violatorias de los derechos de la población adolescente y que imposibilitan la existencia de condiciones para brindar un cuidado integral acorde (Comité de los Derechos del Niño de Uruguay, 2017; Institución Nacional de Derechos Humanos \& Defensoría del Pueblo, 2018).

\section{Posicionarse desde la perspectiva del cuidado}

Los temas de niñez y adolescencia en Uruguay han sido fuertemente estudiados; sin embargo, existen menos antecedentes de investigación vinculados al funcionamiento de la protección social (Leopold, 2014; López-Gallego, 2016; López \& Palummo, 2013; Montes-Maldonado, López-Gallego, \& Galeotti-Galmes, 2018; Morás, 2016) y menos aún de los cuidados de adolescentes en contextos institucionales (Condon \& Pérez, 2014; Rodríguez, 2016).

Los/as adolescentes que cumplen privación de libertad han atravesado en su crecimiento por una precaria organización social de los cuidados (Ávila-Navarrete, 2017; Faur, 2014; Tenenbaum, 2018) que afecta gravemente sus oportunidades de desarrollo; además de incluirlos e incluirlas en una trama institucional estatal destinada a la administración de la infancia-adolescencia pobre con un marcado sesgo disciplinario y punitivo (Llobet, 2012). Las condiciones de recursos insuficientes de las que provienen son atravesadas por una desfavorable situación socioeconómica, referentes familiares en privación de libertad, dificultades para el acceso y permanencia en el sistema educativo formal, entre otros (Morás, 2016). Elementos estos que los/as han expuesto a redes de cuidado deficitarias donde ingresar al sistema penal es una consecuencia posible. 
Los desarrollos sobre cuidados dentro de los estudios de género constituyen un campo extenso de producción teórica con aportes desde diversas perspectivas. Se hallan antecedentes desde los cuidados como trabajo (Carrasco, Borderías, \& Torns, 2011), economía de los cuidados (Pérez-Orozco, 2014), dimensionamientos acerca del uso del tiempo para cuidar (Legarreta, 2014), análisis del social care, regímenes de bienestar y regímenes de género (Daly \& Lewis, 2011; Vera-Rojas, Montes-Maldonado, \& de La BarraUrquieta, 2016), entre otras aproximaciones.

Fisher y Tronto (2013) definen el cuidado como actividades humanas que se realizan para el sostenimiento de la vida entre personas, objetos y el contexto. Implica conflicto e integra relaciones de poder abarcando diferentes momentos y cualidades morales como: atención, responsabilidad, competencia, capacidad de respuesta y confianza.

El artículo aborda la perspectiva del derecho universal a cuidar, ser cuidado (Tronto, 2005) y a participar de relaciones de cuidado que se modifican según necesidades, niveles de dependencia y transformaciones del ciclo de vida. Postulamos al cuidado como dimensión de análisis (Faur, 2014) para comprender la compleja trama que implica cuidar en el contexto institucional de los centros de privación de libertad donde la responsabilidad de protección de los/as adolescentes se halla socavada por el imperativo punitivo.

En función de lo planteado, el artículo analiza los significados y sentidos (Spink, 2013) que le otorgan al cuidado las personas encargadas de los centros de privación de libertad. La identificación de acciones de cuidado y reflexión sobre el cuidado desde su sentido material (mantenimiento de la vida), como simbólico (disposición afectiva para cuidar) se hace con mucha dificultad. Y, por este motivo, es imprescindible la discusión de incluir al cuidado como derecho humano en el contrato social entre ciudadanía, Estado e instituciones (Marrades, 2016).

La perspectiva de estudio de producción de sentidos y prácticas discursivas en lo cotidiano (Spink, 2013, 2014) permite considerar la historicidad de las prácticas, posicionamiento en las relaciones sociales, de poder, negociaciones y conocer las concepciones que orientan la acción y desnaturalizarlas. Aproximarnos a estos significados posibilita conocer cómo se compone la ética del cuidado (Tronto, 2013) producida en el contexto de una institución de encierro con fines de castigo para adolescentes y visibilizarla es un primer paso para realizar modificaciones.

Así las cosas, el objetivo principal del estudio es analizar las prácticas sociales de cuidados en el sistema penal juvenil Uruguayo. Para eso seguimos dos objetivos específicos: 
- Comprender el funcionamiento de las medidas judiciales privativas de libertad.

- Visualizar las concepciones de cuidado que sustenta la intervención.

Las preguntas que guían el estudio son: ¿cómo se componen las prácticas sociales de cuidado en el sistema penal juvenil?, ¿cómo funcionan las medidas judiciales privativas de libertad? y ¿cuáles son las concepciones de cuidado que sustentan la intervención?

\section{Diseño metodológico}

El estudio es cualitativo (Denzin \& Lincoln, 2017) y desarrolla un estudio de caso de tipo único, descriptivo (Yin, 1993), en profundidad (Flyvbejereg, 2004) y con perspectiva etnográfica (Guber, 2013). Se prioriza la perspectiva situada y el análisis de la reflexividad de la investigadora como enfoque de posicionamiento en el escenario de la investigación y relacionamiento con los/as participantes (Montes-Maldonado et al., 2018).

\section{Corpus y participantes}

Se realizó el trabajo de campo durante cuatro meses (entre septiembre y diciembre de 2017), en Montevideo, Uruguay. La totalidad del corpus incluyó actividades realizadas en dos instituciones: Instituto del Niño y Adolescente (Inau) e Inisa. Se compone de 34 entrevistas en profundidad. Además de instancias de observación realizadas en el tránsito por diferentes espacios institucionales y el registro en un diario de campo. En función de los objetivos del presente artículo se utilizan únicamente los materiales del escenario de Inisa integrado por 15 entrevistas en profundidad.

La unidad de análisis son las prácticas de cuidado y, para conocer las mismas, se realizaron entrevistas en profundidad con agentes institucionales que ocupan roles diversos y que tienen contacto directo de forma periódica con la población adolescente privada de libertad. Para la selección de participantes se utilizó un tipo de muestreo no probabilístico intencional y definido por criterios (Ritchie \& Lewis, 2003). La cantidad de entrevistas no fue preestablecida sino que se definió en función de la accesibilidad a los/as participantes y el proceso de saturación de la información cuando los datos empezaron a reiterarse y no apareció información nueva (Ruiz-Olabuénaga, 2012). Los criterios de inclusión muestrales fueron: sexo, edad, rol desempeñado, formación, antigüedad en la tarea y tipo de centro donde se desempeña. Se excluyeron de la selección aquellas personas 
que carecían de contacto directo con adolescentes en el funcionamiento cotidiano de los centros. Así participaron 15 personas que se desempeñan en centros de privación de libertad y semilibertad, 6 varones y 7 mujeres. Respecto a los roles que ocupan en la institución, tres ocupan cargo de educador/a, seis en área psi (psicología, psiquiatría), uno en el área de educación social, tres en trabajo social, uno en el área jurídica y uno posee un cargo de dirección-coordinación. Respecto a los centros de procedencia de los/as participantes se intentó abarcar la mayor diversidad posible, accediendo a los siguientes: ingreso, estudio y derivación; centros de adolescentes varones entre 15-17; máxima contención; adolescentes mujeres; adolescentes entre 12-14 años y un centro de semilibertad.

La validez del estudio radica en la consulta a la mayor diversidad posible entre los informantes-participantes que ocupan los diversos roles en distintos espacios de la institución. Esto posibilita acceder a diferentes posicionamientos y versiones sobre las prácticas de cuidado (Ruiz-Olabuénaga, 2012).

\section{Análisis}

El análisis de la información sigue el procedimiento de análisis temático cualitativo (Spencer, Ritchie, O'Connor, Morrell, \& Ormston, 2014). El mismo contiene cinco etapas diferenciadas: familiarización con el conjunto de datos; construcción de un marco temático inicial; indexación y clasificación de los datos; revisión de los segmentas clasificados y refinamiento del marco temático y resumen de los datos y visualización de los mismos.

Para la codificación, segmentación y recuperación de datos en las fases analítica e informativa de la investigación se utilizó el software de análisis de datos cualitativos asistido (Caqdas), MaxQda (v. 2018).

\section{Resguardos éticos}

La investigación está avalada por el Comité de Ética de la Facultad de Psicología (Universidad de la República) y cuenta con las autorizaciones correspondientes para realizar el estudio en las instituciones públicas involucradas. El procedimiento sigue las exigencias éticas para el uso de la información de modo que preserva la confidencialidad, privacidad y anonimato de las personas participantes utilizando codificación. Además, utiliza consentimiento informado firmado respetando la autonomía y voluntariedad de la participación en el estudio (American Psychological Association, 2017). 


\section{Resultados}

Se detallan las reflexiones que surgen respecto a la noción de cuidados en las entrevistas en profundidad mantenidas con los/as participantes de la investigación.

La reflexión sobre los cuidados no se encuentra extendida en el discurso cotidiano de los/as agentes de la institución, ni planteado como objetivo a seguir. De alguna manera aparece a veces como categoría omitida o como algo que realizan sin tenerlo conceptualizado o identificado. Por tanto, reflexionar sobre cómo cuidan en el contexto de encierro fue una propuesta en las entrevistas donde se indagó al respecto y se facilitó la discusión y reflexión a través del intercambio para así pensar en la gestión del cuidado, las acciones que implementan, cómo lo comprenden y con qué limitaciones se encuentran. El análisis que se presenta da cuenta de una categoría inestable, plena de tensiones y contradicciones en cuanto a sus significados que las vamos a desmenuzar en tres líneas analíticas.

\section{Acerca de la medida privativa de libertad y sus objetivos}

El marco de legitimidad para la ejecución de esta medida judicial está dado por la legislación nacional e internacional vigente. El CNA (Uruguay, 2004) expone que la privación de libertad «consiste en recluir al adolescente en un establecimiento que asegure su permanencia en el recinto, sin menoscabo de los derechos consagrados en este Código, las normas constitucionales, legales e instrumentos internacionales».

Por su parte la ley de creación de Inisa (Uruguay, 2015) tiene por objetivo

la inserción social y comunitaria de los adolescentes en conflicto con la ley penal mediante un proceso psicosocial, educativo e integral, que conlleve el reconocimiento de su condición de sujetos de derecho» y que sus cometidos integran «el interés superior del adolescente y en estricta observancia de la normativa nacional e internacional en la materia, lo que se expresa en el reconocimiento, respeto y garantía de sus derechos.

El escenario institucional de ejecución de las medidas es objeto de reprobaciones permanentes y señalado como en constante situación crítica. Las citadas leyes respecto a los objetivos institucionales expresan una gran ambigüedad en sus fines, lo cual deja espacio libre para el accionar discrecional, sumado a que las definiciones sobre el conteni- 
do de las medidas socioeducativas han sido históricamente fuente de debates. Polémica que no se encuentra saldada por parte de los mandos ejecutivos ni tampoco desde el discurso del personal que trabaja diariamente con los/as adolescentes. Se puede ver en las citas de entrevistas referencias a los fines de la medida que la asocian con objetivos de, castigo, responsabilización por la infracción cometida o el daño, educación/reeducación, inserción/reinserción social, rehabilitación, entre otras.

En general uno sabe que las cárceles no rehabilitan, eso está bastante estudiado: de que este formato no. Capaz si se actuara mucho más desde la prevención, o cuando, por ejemplo, criminológicamente se puede hacer una diferenciación; este joven, por ejemplo, trabajarlo desde otro lugar, desde el afuera, me parece que sería mucho más positivo. Entonces generalizar me parece que no es posible en esto, porque si bien hay determinadas cosas que son un poco más constantes habría que analizar caso por caso, y esa parte es la que me parece que falla también, ¿no? Se termina generalizando una medida para todos, y puede ser que en algunos casos les aporte algo a los jóvenes, puede ser. También eso me parece que siempre lo que la privación de libertad puede aportar es algo bastante efímero, que a la larga me parece que es peor también. (E8, hombre, área psi.)

La institución me pide cosas totalmente contradictorias, a la gente que trabaja, la institución te pide que no haya fuga, que la seguridad y te pide la inserción, y que los gurices [adolescentes] tengan una medida socioeducativa, pero, «Te voy a hacer una propuesta mira: quiero que Fulanito salga a no sé qué», dependerá del centro, de cuánto esté enfocado a las medidas de seguridad.

-¿Qué se entiende por inserción?

-No, acá en esta institución para mí no se entiende; es como una palabra vacía de contenido acá. (Eg, mujer, educadora)

Para mí el sistema es malo, la idea desde el afuera es; una medida socioeducativa, que se formen, que generen hábitos, que tengan una formación educativa prelaboral para después insertarse, etcétera. Todo muy lindo, pero después en los hechos acá la formación no es buena, hay un montón de problemas, es insuficiente en todo sentido y bueno, el sistema funciona mal, el sistema no es bueno. Para mí lo único bueno que tiene esto es que genera un parate en los jóvenes, que vienen a veces desbarrancando mal en el tema de adicciones o delinquiendo, que pueden terminar muertos. (E10, mujer, trabajadora social)

Las estrategias para ejecutar la medida dependen y varían en cada centro y estilo de los equipos de trabajo. Las últimas legislaciones sumadas a la presión de la opinión pública sobre la incidencia de la infracción de adolescentes en la seguridad pública generan 
un clima de argumentación y justificación que erige al encierro como la principal solución posible para la transgresión. Pero no cualquier encierro sino aquel que se produce en determinadas condiciones de precariedad, sufrimiento, privaciones múltiples y total aislamiento social. Cabe destacar aquí la presencia de sectores de la sociedad civil y movimientos sociales que han realizado acciones que van a contracorriente de esta perspectiva.

El castigo (Baratta, 2004; Foucault, 2002; Garland, 2006) como una función social compleja responde a valoraciones morales y culturales. Foucault (2002) habla del suplemento punitivo, como aquello que excede la privación de libertad, que va más allá de sus fines explícitos, donde además de perder la libertad se castiga a través de las condiciones del encierro y rituales de producción de sufrimiento. En esta misma línea López y Daroqui (2012) hablan del castigo dentro del castigo, y detallan las prácticas punitivas violentas como pueden ser las requisas, utilización de esposas y grilletes, agresiones físicas o verbales, entre otras vulneraciones.

Las acciones de cuidado posibles en el encierro son discrecionales, improvisadas y voluntarias, su fin principal se vincula con el mantenimiento de la vida en sus aspectos más imprescindibles dejando de lado la respuesta integral de cuidado.

\section{La seguridad como criterio de funcionamiento y legitimación de violencia}

En el funcionamiento cotidiano de los centros se destaca la idea de seguridad. La misma opera como principio de organización, criterio de funcionamiento y legitimación del uso de prácticas violentas. Además, integra diferentes aspectos según explican los/as entrevistados/as. Se relaciona con evitar fugas, prevenir motines, evitar daños físicos entre ellos/as, hacia sí mismos/as o hacia funcionarios/as. En suma, mantener el orden general del centro en cuanto al respeto de horarios, rutinas y actividades y cuidar de la integridad física. Así explica una educadora social:

El cuidado es muy difícil porque siempre te gana la seguridad, siempre te gana la seguridad. A mí me costó un montón adaptarme a eso.

Primero es la seguridad del gurí [adolescente] y después... Pero por suerte (yo te puedo hablar por este centro que es lo que veo) el cuidado está muy a la par; el cuidado, la contención, el acompañamiento del gurí [adolescente] está muy a la par. A ver, siempre el tema de la seguridad y el tema de que bueno, ya la requisa es violenta, los gurices [adolescentes] hay veces que te dicen «Ay por favor, me requisé hoy diez veces» porque claro, fue 
al dentista se requisó ida y vuelta, fue a psicoterapia y se requisó ida y vuelta, fue al taller y se requisó ida y vuelta, fue al liceo y se requisó ida y vuelta, y después si le duele la cabeza y va al policlínico se requisa ida y vuelta; entonces eso es violento y lamentablemente nosotros tendemos a acostumbrarnos a esa violencia. (E12-Mujer-Educadora Social)

Con la excusa de mantener el orden interno de los centros se justifica con motivos de seguridad prácticas que se pueden significar como crueles y despiadadas hacia los/as adolescentes. Un tratamiento que se aleja de la supuesta especialidad de la justicia juvenil.

Los centros presentan características cada vez más similares a los lugares de reclusión de adultos como grandes complejos penitenciarios con celdas, rejas, traslados entre centros como forma de sanción o penitencia, requisas, uso de grilletes y esposas, entre otras prácticas de control y sujeción. Algunos/as entrevistados/as destacan esta situación como un alejamiento del objetivo de educación como fin del tratamiento de los/as adolescentes que pone por principal objetivo la seguridad y justifica acciones violentas. Así explican:

En el caso de los adolescentes, siempre se trabajó diferente. La regla, el límite que es la seguridad viene junto con lo educativo. El mismo que le pone la esposa y que de pronto lo tiene que contener porque está en un desborde y lo tenés que agarrar y sujetar, es después el que se sienta y habla con el chiquilín [adolescente] de lo que pasó, o le lleva la taza de café con leche. Ese límite y lo educativo van de la mano, los psicólogos lo sabemos. Le ponés el límite al niño y al mismo tiempo le das amor, en esto en un momento fue así, pero ella [la nueva administración] creó un grupo que es como una especie de grupo Geo que interviene; es como una policía, como unos parapoliciales. Arrancamos de una base que para mí está mal, cuando separás límite de afecto, ya arrancamos mal, es violencia. (E7, mujer, área psi.)

I: - ¿Cuándo entran los revisan?

E1: -Sí. Y los desnudan totalmente, los revisan todos.

- ¿Eso es tarea de quién?

-Es del equipo de seguridad.

- ¿Pero no son policías?

-No, no son policías.

- ¿Son educadores?

- No, son de seguridad.

- ¿Y los revisan cuando entran y cuando se van? 
-No, solo cuando vienen para que no ingresen. Yo que sé, en el imaginario posible puede haber desde drogas, armas, objetos. (E1, hombre, educador)

Requisas, uso de esposas y grilletes y medios de contención física son prácticas habituales en los centros. Desde 2017 cuentan con protocolos que establecen la normativa para la realización de estas tareas que denominan Reglamento disciplinario: infracciones de las normas de convivencia (Inisa, 2017a) y Reglamento provisorio del procedimiento de contención física de adolescentes privados de libertad en situación de conflicto (Inisa, 2017b). Se explicita en estos documentos detalles que permiten visualizar los fuertes regímenes disciplinarios a los que están expuestos los/as adolescentes y la primacía del criterio de seguridad para el funcionamiento del encierro. La seguridad se torna un componente central de la ética de cuidado imperante en la gestión de los centros.

Es importante destacar estudios que muestran resultados negativos, nulos e incluso efectos negativos del tratamiento de adolescentes en regímenes cerrados, donde se prioriza impartir disciplina y punir (Morás, 2016; Redondo et al., 2012). Sin embargo, los programas de tratamiento que priorizan las medidas de tipo comunitarias sí destacan resultados positivos (Redondo \& Martínez, 2013).

\section{Acceso a los derechos a través de la política punitiva}

La retórica sobre los derechos de niñez y adolescencia tiene gran presencia en los centros, aunque esto no sucede sin conflictos o negociaciones de sentido. Las referencias a los derechos humanos de adolescentes surgen como enfoque de trabajo o intervención, como ideal a seguir o algo a proyectar, para describir la situación de adolescentes en función del mapeo del acceso a los derechos y también para pensar posibles formas de restitución de derechos vulnerados.

En algunas entrevistas se visualizan trayectorias de vulneración de derechos de adolescentes como falta de alimentación, higiene, estrategias de vida en calle y así plantean que la medida judicial es una oportunidad para lograr satisfacer algunas necesidades básicas de protección o recibir desde alimentos hasta atención médica u otros recursos.

También muestran como la población adolescente utiliza la infracción como medio de autocuidado y vínculo con el mundo institucional, dado que los derechos son universales, pero no todas las personas acceden de igual manera. Lo cual expone las dificultades en las trayectorias de algunos/as adolescentes de acceder a los derechos y satisfacer nece- 
sidades y cómo el Estado funciona como productor de desigualdades en función de los procesos de inclusión y exclusión social a los derechos (Medan et al., 2019).

Desde las entrevistas lo argumentan de la siguiente manera:

(...) por ejemplo hay gurisas [adolescente] que lo toman como una oportunidad, pero no con respecto a la infracción, eso habla de que tenemos un sistema de protección tan terrible que una gurisa tiene que cometer una infracción para autocuidarse. Para autocuidarse porque ingresaba a [Centro de la institución de protección] y le daban los boletos para que vuelva a la casa. Entonces, por otra cuestión terminó siendo oportunidad y muy perversamente. $Y$ opera muchas veces como alternativa a la protección (E9, mujer, educadora).

Hace 9 años que trabajo en la institución. Cuando ingresa el joven haces asistencialismo, le das todo. Afuera vuelve a lo mismo, al mismo contexto. En el egreso no tiene educadores que le digan levántate a tomar la leche, ese es el problema. En su casa están con las manos vacías (E14, mujer, área psi).

Acá la pasan bomba, comen las cuatro comidas, salen sanos, tienen dentista, a los que le faltan los dientes se le ponen las piezas, tienen psiquiatra, están atendidos. (E2, hombre, área psi)

Las características definidas por Goffman (2001) para explicar las instituciones totales pueden verse reflejadas en este contexto donde todos los aspectos de la vida se realizan en la institución, hay una organización del tiempo y espacio monótono y tedioso, homogenización de las necesidades y formas de satisfacer las mismas desde lo burocrático, separación de los vínculos familiares, sociales y comunitarios. El tránsito de los/as adolescentes se encuentra vigilado y controlado por el personal de la institución lo que lleva a la existencia de dos grupos de personas bien diferenciados en cuanto a estatus, roles y funciones. Ambos grupos marcados por la asimetría de poder establecen elementos estereotipadas del otro/a para mantener la distancia y diferenciarse: adolescentes y personal del centro.

En este marco vemos cómo se despliegan algunas lógicas de interacción particular como, por ejemplo, el sistema de premios y castigos en función de la adherencia o no a las normas de comportamiento establecidas o las normas no escritas e infrapenales (Foucault, 2002). Los traslados de un centro a otro caracterizados como el mejor o peor centro en función de su nivel de encierro y actividades o alimentación pueden oficiar de premio o castigo según la situación. De igual manera funciona con el acceso a programas educativos o laborales donde algunos/as pueden ser designados o no. 
Si el gurí [adolescente] está demostrando que quiere ir al liceo, que va todos los días, que guarda sus cuadernitos, que se ocupa de sus cosas. Que no hay que ni despertarlo, ese es elegido. Hay una selección natural en esto, y está en la voluntad de ellos. Pero la selección tiene que ver con la voluntad de ellos. Despertarle el estímulo, estimularlos, es nuestra tarea de técnicos y de funcionarios. Pero la matriz no es la misma en todas y tenemos 570 individualidades acá adentro. (E2, hombre, área psi)

La propia biografía o ciertas vivencias de vulneración de derechos en la historia de vida también se constituyen en vehículos de acceso a determinadas oportunidades o como valor de cambio. La disposición a contar estas historias una y otra vez ante el equipo técnico, mostrar voluntad de cambio o esfuerzo son elementos que se valoran positivamente en las valoraciones técnicas.

Cuando hay chiquilines [adolescentes] que son muy sumisos, que son buenos presos generan empatía en los educadores y ahí sí se conectan con las historias de los chiquilines, intentan hacer algo, se transforman en referencias. (...) hay educadores que les llevan cosas de su casa: ropa, tabaco, cuando no reciben visitas. Se da eso, y eso como muy del cuidado cuando no está la familia, cuando no están en la comunidad, cuando no hay nadie afuera que pueda acompañar el encierro. (E9, mujer, educadora)

Esa anotación policial los hizo llegar a otro [servicio de atención] ¿entendés? Salado, que te digan que la protección social llegó... Te lo digo como algo para cuestionarlo como nada, como Estado. (E15, mujer, directora)

Merklen (2013) habla de políticas de individuación para explicar las nuevas relaciones entre Estado y sociedad y la reorientación de la política centrada en la producción del individuo, por medio de las cuales se emprende la creación de nuevos dispositivos que persiguen objetivos de responsabilización y activación. Estas exigencias dirigidas sobre las personas buscan que cada quién se haga cargo de su suerte y de los avatares de la vida en sociedad. Con lo cual para asumir riesgos y asegurarnos de los mismos se hace necesaria la activación de la voluntad individual. Las políticas del individuo conllevan nuevos métodos de intervención social dejando atrás un modelo tutelar a partir de la búsqueda de autonomía y de políticas que abogan por el empoderamiento y orientación para impulsar al individuo a cambiar sus condiciones de vida. Estos dispositivos se centran en el examen subjetivo de los/as participantes para generar mecanismos de inclusión-exclusión de los beneficios de la política a partir de hechos biográficos. 
En nuestro caso de análisis podemos observar ciertas argumentaciones asociadas a lógicas de favor, caridad o compasión. Así como también la tendencia a responsabilizar a los/as adolescentes, sus familias o su espacio social de pertenencia (barrios calificados de peligrosos, grupos de pares) como responsables de su situación. Interesa además destacar el proceso por el cual estos/as adolescentes logran alcanzar determinados derechos sociales u obtener recursos a partir de su paso por la política punitiva. Esto evidencia las falencias de las políticas de protección y la tensión de observar lógicas y objetivos similares entre el sistema de bienestar y de control social.

\section{Conclusiones}

El artículo analiza las prácticas de cuidado presentes en la gestión de los centros de privación de libertad para adolescentes e intenta comprender cómo funciona la medida judicial y cómo conciben al cuidado. Esta reflexión expone las negociaciones de sentido, normas y valores para realizar esta tarea y acercarnos a comprender la ética de cuidado imperante.

En primer lugar, vemos que la medida privativa de libertad es ambigua y poco específica en su definición, lo cual deja espacio libre para el desarrollo de estrategias discrecionales, improvisadas y donde priman las voluntades individuales para llevar adelante el objetivo de restitución de los derechos vulnerados y, en este camino, también poder cuidar. Aspectos que se relacionan también con las formas de tratamiento de la penalidad juvenil a nivel regional (Ávila-Navarrete, 2017; Cienfuegos, 2015; Medan et al., 2019; Leal \& Macedo, 2019).

La gestión del cuidado se encuentra contrapuesta a la gestión del encierro donde para poder mantenerlo se hace necesario postular la seguridad como un elemento fundamental del funcionamiento y en esta línea las acciones para mantenerla avasallan las posibilidades de brindar un cuidado integral. La seguridad aparece en el centro de la gestión del funcionamiento cotidiano sobre el cual se organiza la vida de los/as adolescentes. El objetivo de mantener el orden y evitar las fugas genera que se legitimen todo tipo de prácticas de control y vigilancia que son crueles y violentas como el uso de grilletes o esposas.

Por último, mostramos como muchos/as adolescentes logran acceder a derechos recién cuando son objetos de política punitiva. Es decir, el tránsito por el sistema penal se 
les presenta como una oportunidad de goce de derechos a los que antes no pudieron acceder por sus condiciones de vida precarias. Hecho que además da cuenta de la producción de desigualdades en los procesos de inclusión-exclusión social por parte del Estado y los abordajes burocrático-institucionales (Medan et al., 2019).

El personal de los centros de privación de libertad en Uruguay da viabilidad a una determinada forma de castigo por la vía del encierro disciplinante que legitima el uso de la violencia según corresponda y este elemento transversaliza la ética del cuidado. De todas maneras, esto no sucede sin cuestionamientos ni de forma homogénea dado que coexisten en la institución diversas opiniones y formas de hacer, sentimientos y creencias encontradas que le otorgan multiplicidad.

Se actualiza la pregunta de Foucault (2016) sobre cómo se gesta, acepta y generaliza la utilización de la prisión como forma de castigo y en este caso, podemos agregar, cómo se hace aceptable esta forma de tratamiento para adolescentes. Medan (2017) explica que coexisten dos formas de percibir a los/as adolescentes-jóvenes que transitan entre la plena responsabilidad por las infracciones cometidas y las necesidades de protección, en una lucha entre el interés superior del/a adolescente que pierde fuerza en pos del interés por la seguridad de la población.

\section{Limitaciones y desafíos}

Como limitaciones del estudio destacan las dificultades de accesibilidad a las instituciones de encierro y de coordinación tanto para el ingreso, tránsito y acceso a la información. Si bien las personas participantes de la investigación son acotadas, permite conocer y analizar las prácticas de cuidado en los centros de privación de libertad para adolescentes. De todas formas, sería importante acceder a una mayor cantidad de personal y centros, así como también conocer la perspectiva de las personas que ocupan altos mandos donde se define y gestiona la política.

El desafío de futuro será modificar la ética de cuidado (Tronto, 2013) dominante y construir un camino alternativo donde se postule una concepción de cuidados integral como perspectiva para transversalizar la planificación de políticas, el accionar cotidiano en los centros, un modelo de evaluación de implementación de las medidas judiciales y se constituya también como un ideal a seguir. Asimismo, redefinir el tratamiento actual que reciben los/as adolescentes y hacer énfasis en el aspecto de responsabilidad de cuidado que tiene la sociedad en general y esta institución en particular de restituir las 
condiciones de cuidado y construir un sistema penal que garantice cuidar desde los aspectos materiales y simbólicos.

\section{Agradecimientos}

Al Instituto Nacional de Inclusión Social Adolescente por permitir la investigación y todas las personas que colaboraron en las entrevistas. A las directoras de tesis, Dra. Encarna Bodelón y Dra. Estibaliz de Miguel, por los comentarios sobre el artículo.

\section{Referencias}

American Psychological Association. (2017). Ethical principles of psychologists and code of conduct. Washington, D. C.: Autor.

Ávila-Navarrete, V. (2017). ¿Corresponsabilidad familiar en instituciones de reeducación para adolescentes infractores? Revista Latinoamericana de Ciencias Sociales, Niñez y Juventud, 15(2), 1191-1206. https://doi.org/10.1160o/1692715x.1115080812

Baratta, A. (2004). Criminología crítica y crítica del derecho penal: introducción a la sociología jurídico penal. Buenos Aires: Siglo XXI.

Carrasco, C., Borderías, C., \& Torns, T. (2011). El trabajo de cuidados: antecedentes históricos y debates actuales. En C. Carrasco, C. Borderías, \& T. Torns (Eds.), El trabajo de cuidados: historia, teoría y políticas (pp. 13-96). Madrid: Catarata.

Cienfuegos, A. (2015). Lo político y las políticas de la delincuencia juvenil: una reflexión sobre el caso de jóvenes vinculados al Sistema de Responsabilidad Penal Adolescente. De prácticas y discursos: Cuadernos de Ciencias Sociales. 4, 1-18. Recuperado de: http://depracticasydiscursos.unne.edu.ar/Revista4/index.html

Comité de los Derechos del Niño de Uruguay. (2017). Situación general de los derechos de niños, niñas y adolescentes. Informe de Diagnóstico. Montevideo: Comité de los Derechos del Niño de Uruguay. Recuperado de: http://www.comitednu.org/wpcontent/uploads/2014/o8/Informe-diagnostico-2017.pdf

Condon, F., \& Pérez, C. (2014). Niñas, niños y adolescentes privados del cuidado de sus familias o en riesgo de estarlo en las políticas de cuidado. Montevideo: Mastergraf.

Daly, M., \& Lewis, J. (2011). El concepto de «social care» y el análisis de los Estados de Bienestar contemporáneos. En C. Carrasco, C. Borderías, \& T. Torns (Eds.), El trabajo de cuidados: historia, teoría y políticas (pp. 225-251). Madrid: Catarata. 
Denzin, N., \& Lincoln, I. (2017). The Sage handbook of qualitative research. Londres: Sage.

Fassin, D. (2018). Castigar. Buenos Aires: Adriana Hidalgo Editora.

Faur, E. (2014). El cuidado infantil en el siglo XXI: mujeres malabaristas en una sociedad desigual. Buenos Aires: Siglo XXI.

Flyvbejereg, B. (2004). Cinco malentendidos acerca de la investigación mediante los estudios de caso. Reis, 106(4), 33-62. https://doi.org/10.2307/40184584

Foucault, M. (2002). Vigilar y castigar: nacimiento de la prisión. Buenos Aires: Siglo XXI.

Foucault, M. (2016). La sociedad punitiva: curso en el Collége de France (1972-1973). Buenos Aires: Fondo de Cultura Económica.

García, S. (2010). Políticas de protección especial. Montevideo: Enia.

Garland, D. (2006). Castigo y sociedad moderna: un estudio de teoría social. Ciudad de México: Siglo XXI.

Garland, D. (2018). Theoretical advances and problems in the sociology of punishment. Punishment \& Society, 20(1), 8-33. https://doi.org/10.1177/1462474517737274

Goffman, I. (2001). Internados: ensayos sobre la situación social de los enfermos mentales. Buenos Aires: Amorrortu.

González, C. (2015). La infracción adolescente a la ley penal en el centro del debate sobre seguridad pública en Uruguay. Revista Latinoamericana de Ciencias Sociales, Niñez y Juventud, 2(13), 951-962. https://doi.org/10.1160o/1692715x.13228201014

González, C. (2016). Modelos de justicia penal juvenil en debate a inicios del siglo XXI. Quaestio Iuris, 2(9), 652-669. https://doi.org/10.12957/rqi.2016.18008

Guber, R. (2013). La articulación etnográfica: descubrimiento y trabajo de campo en la investigación de Esther Hermitte. Buenos Aires: Biblos.

Inisa. (2017a). Reglamento disciplinario: infracciones de las normas de convivencia. Montevideo: ONU.

Inisa. (2017b). Reglamento provisorio del procedimiento de contención física de adolescentes privados de libertad en situación de conflicto. Montevideo: ONU.

Institución Nacional de Derechos Humanos, \& Defensoría del Pueblo. (2018). VI Informe Anual de la Asamblea General 2017. Montevideo: Mastergraf.

Laurnaga, M. (2007). Mesa de diálogo Políticas de inclusión social: análisis y propuestas. En C. Fassler (Comp.), Red de género y familia (pp. 23-35). Montevideo: Trilce.

Leal, D., \& Macedo, J. (2019). Os discursos protetivos e punitivos acerca dos adolescentes em medida de internação no Brasil. Revista Latinoamericana de Ciencias Sociales, Niñez y Juventud, 17(1), 207-221. https://doi.org/10.1160o/1692715x.17112 
Legarreta, M. (2014). Cuidados y sostenibilidad de la vida: una reflexión a partir de las políticas de tiempo. Papeles del Ceic, 1(104), 1-36. https://doi.org/10.1387/pceic.12427 Leopold, S. (2014). Los laberintos de la infancia: discursos, representaciones y crítica. Montevideo: Csic.

López, A., \& Daroqui, A. (2012). Castigos dentro del castigo. En A. Daroqui, A. López, \& R. Cipriano (Eds.), Sujeto de castigos: hacia una sociología de la penalidad juvenil (pp. 303-321). Rosario: Homo Sapiens.

López, A., \& Palummo, J. (2013). Internados: las prácticas judiciales de institucionalización por protección de niños, niñas y adolescentes en la ciudad de Montevideo. Montevideo: Unicef.

López-Gallego, L. (2016). Prácticas de control socio-penal: dispositivo psipericial y adolescentes mujeres en el Sistema Penal Juvenil Uruguayo. (Tesis Doctoral). Barcelona: Universidad Autónoma de Barcelona.

Llobet, V. (2012). Políticas sociales y ciudadanía. Diálogos entre la teoría feminista y el campo de estudios de infancia. Frontera Norte, 24(48), 7-36.

Marrades, A. (2016). Los nuevos derechos sociales: el derecho al cuidado como fundamento del pacto constitucional. Revista de Derecho Político, 97, 209-242. https://doi.org/10.5944/rdp.97.2016.17623

Medan, M. (2017). El gobierno de la juventud en riesgo en el Amba: entre la seguridad y la inclusión. Buenos Aires: Teseo Press.

Medan, M., Villalta, C., \& Llobet, V. (2019). Entre inercias burocráticas y evaluaciones sobre las familias: adolescentes privados de libertad en Buenos Aires, Argentina. Estudios Socio-Jurídicos, 21(1), 293-326. https://doi.org/10.12804/ revistas.urosario.edu.co/sociojuridicos/a.6309

Merklen, D. (2013). Las dinámicas contemporáneas de la individuación. En R. Castel, G. Kessler, D. Merklen, \& N. Murard (Eds.), Individuación, precariedad, inseguridad: ¿desinstitucionalización del presente? (pp. 45-86). Buenos Aires: Paidós.

Montes-Maldonado, C., López-Gallego, L., \& Galeotti-Galmes, R. (2018). Adolescentes mujeres y medidas no privativas de libertad: narrativas de una experiencia etnográfica. Psicoperspectivas, 17(2), 1-12. https://doi.org/10.5027/psicoperspectivasvoli7-issue2-fulltext-1212

Morás, L. E. (2016). Estudio de las trayectorias de vida de adolescentes en conflicto con la ley con particular énfasis en la relación delito-trabajo. Montevideo: OIT, Cinterfor.

Pérez-Orozco, A. (2014). Subversión feminista de la economía: aportes para un debate sobre el conflicto capital-vida. Madrid: Traficantes de Sueños. 
Redondo, S., Martínez, A., \& Pueyo, A. (2012). Intervenciones con delincuentes juveniles en el marco de la justicia: intervención y aplicaciones. EduPsykhé. Revista de Psicología y Educación, 11(2), 143-169.

Redondo, S., \& Martínez, A. (2013). Evaluación criminológica de la justicia juvenil en España. Cuadernos de Política Criminal, 2(110), 189-220.

Ritchie, J., \& Lewis, J. (Eds.). (2003). Qualitative research practice: A guide for social science students and researchers. London: Sage.

Rodríguez, C. (2016). Lo insoportable en las instituciones de protección a la infancia. Montevideo: Azafrán.

Ruiz-Olabuénaga, J. (2012). Metodología de la investigación cualitativa. Bilbao: Deusto. Spencer, L., Ritchie, J., O'Connor, W., Morrell, G., \& Ormston, R. (2014). Analysis in practice. En J. Ritchie, J. Lewis, C. McNaughton, \& R. Ormston (Eds.), Qualitative research practice: A guide for social science students and researchers (pp. 295-345). Londres: Sage.

Spink, M. J. (2013). Práticas discursivas e produção de sentidos no cotidiano. Río de Janeiro: Edelstein.

Spink, M. J. (2014). Viver em áreas de risco: tensões entre gestão de desastres ambientais e os sentidos de risco no cotidiano. Ciênc. saúde coletiva, 19(9), 3743-3754. http://dx.doi.org/10.1590/1413-81232014199.01182014

Tenenbaum, G. (2017). Castigo y capitalismo: la corrupción judicial en el mercado de castigo para adolescentes en conflicto con la ley de la Ciudad de México. Espacio Abierto Cuaderno Venezolano de Sociología 26(1), 69-89. Recuperado de: https://dialnet.unirioja.es/servlet/articulo?codigo $=5910476$

Tenenbaum, G. (2018). Violencia juvenil, familias y calles: ¿dónde se «rescatan» los adolescentes de Montevideo en conflicto con la ley? Revista de Ciencias Sociales, 31(42), 151-175. https://doi.org/10.26489/rvs.v30i41.7

Tronto, J. (2005). Cuando la ciudadanía se cuida: una paradoja neoliberal del bienestar y la desigualdad. En Congreso Internacional Sare 2004 (Eds.), Memorias (pp. 231-253). Vitoria-Gasteiz: Emakunde.

Tronto, J. (2013). Caring democracy: Markets, equality, and justice. Nueva York: University Press.

Uruguay. (1990). Ley nº 16137 de 28 de septiembre de 199o. Convención sobre los Derechos del Niño. Montevideo: Parlamento de Uruguay.

Uruguay. (2004). Ley n⿳ํㅡ 1723 de 7 de septiembre de 2004. Código del Niñez y la Adolescencia. Montevideo: Parlamento de Uruguay. 
Uruguay. (2015). Ley nº 19367 de 31 de diciembre de 2015. Creación del Instituto Nacional de Inclusión Social Adolescente como servicio descentralizado. Montevideo: Parlamento de Uruguay.

Vera-Rojas, W., Montes-Maldonado, C., \& de La Barra-Urquieta, C. (2016). Los cuidados en infancia: regímenes y arreglos familiares en Chile y Uruguay. Psicoperspectivas, 15(3), 34-45. https://doi.org/10.5027/psicoperspectivas-vol15-issue3-fulltext-828

Yin, R. (1993). Applications of case study research. Londres: Sage. 